\title{
Resenha
}

\section{The chance is necessary? The case of The Drunkard's Walk: How Randomness Rules Our Lives}

\author{
Rodrigo Siqueira-Batista ${ }^{1,2}$ e José Abdalla Helayël-Neto² \\ ${ }^{1}$ Departamento de Medicina e Enfermagem, Universidade Federal de Viçosa, Viçosa, MG, Brasil \\ ${ }^{2}$ Centro Brasileiro de Pesquisas Físicas, Rio de Janeiro, RJ, Brasil
}

L. Mlodinow, The Drunkard's Walk: How Randomness Rules Our Lives (Pantheon Books, New York, 2008), $272 \mathrm{p}$.

The debate involving chance and necessity is one of the oldest questions in the history of philosophy. In fact, the mention of the need can be sought in the earlier text of philosophical thought, the fragment left over from Anaximander. However, the long history of epistemological discussions do not overshadow the relevance of the topic, which has been expressed in various contemporary publications, among which stands out The Drunkard's Walk: How Randomness Rules Our Lives, a book by physicist Leonard Mlodinow, currently professor at the Institute Technology California.

The work, as announced in the prologue, which deals with the principles governing the design, highlighting its importance in the fields of politics, business, medicine, economics, sport and leisure, among others. The ten chapters are structured in an orderly fashion deterministically, not by chance - covering 1) the hidden role of chance (Ch. 1 - Peering through the eyepiece of randomness), (2) the basic principles of probability (Ch. 2 - The laws of truths and half-truths), (3) the framework for thinking about random situations (Ch. 3 - Finding your way through a space of possibilities), (4) How to count the number of ways in which events can happen (Ch. 4 - Tracking the pathways to success), (5) the extent to which probabilities are reflected in the results we observe (Ch. 5 - The dueling laws of large and small numbers), (6) how to adjust expectations in light of past events or new knowledge (Ch. $6-$ False positives and positive fallacies), (7) The meaning and lack of meaning in measurements (Ch. 7 - Measure- ment and the law of errors), (8) how large numbers can wash out the disorder of randomness (Ch. $8-$ The order in chaos), (9) why we are often fooled by the regularities in chance events (Ch. 9 - Illusions of patterns and patterns of illusion) e (10) why chance is a more fundamental conception than causality (Ch. 10 - The drunkard's walk).

Although extremely well-written and the efforts in presenting a series of arguments to try to demonstrate the centrality of chance, The Drunkard's Walk approaches this concept only as a matter of knowledge. For example, the proposition that "determinism is a poor model for the human experience" or the affirmation of "our assessment of the world would be quite different if all our judgments could be insulated from expectation and based only on relevant data". The book does not actually tackle this question, which, perhaps, is the center of the debate: is chance intrinsic to the world in ontological terms - or is it dependent on the way it presents itself - from an epistemological viewpoint?[1] In the latter case, would there be an illusion - a result of the impossibility that Homo sapiens sapiens fully understands the world - or, also an insurmountable measure of the human ignorance? Despite the efforts of the author, these ancient questions remain; therefore, it does not really seem to be possible to play God, or not, data...

\section{Referências}

[1] R. Siqueira-Batista e F.R. Schramm, Ciên Saúde Col 13, 207 (2008).

\footnotetext{
${ }^{1}$ E-mail: rsiqueirabatista@yahoo.com.br. 